\title{
Immune-checkpoints Screening in Tumor Specimens of Gastric Cancer from Hospital Biobank in Shanghai
}

\author{
Yingyan Yu, Xia Huang and Zhenggang Zhu. Shanghai Ruijin Hospital, Shanghai Jiao \\ Tong University School of Medicine, 200025, Shanghai, China. e-mail: ruiiinhospitalyyy@163.com
}

Gastric cancer (GC) is one of the indications of FDA approved immune-checkpoint inhibitor, but lack of systematic research for screening indication of targeted therapy. On the basis of T-cell receptor (TCR) signaling pathway analysis on RNA-seq from 277 GC patients of TCGA cohort, a set of T cell markers for predicting sensitivity to immune-checkpoint inhibitor was found. The correlation of genes expression with response to immune-checkpoint inhibitor was evaluated by data in GEO database. The gene expressing status of T-cell markers and the association with the molecular classification in Chinese GC cohort was examined on 579 cases of GC stored in hospital Biobank. EBER in situ hybridization was used for Epstein-Bar virus (EBV) detection, and MLH1 and MSH2 expression was detected by immunohistochemistry for microsatellite instability (MSI) analysis. As results, according to expressing levels of T-cell receptor signaling pathway associated genes, the hospital GC could be divided into TCR signal enrichment high (TCRSE-H) and TCR signal enrichment low (TCRSE-L). The T-cell markers of CD3, CD4, CD8, PD-1, PD-L1, IDO1, LAG3, TIM3 and FOXP3 revealed increased expression in GC tissues than that in normal mucosa. A four-T cell signature covered CD3, CD4, CD8 and PD-1 could be used as an index to discriminate TCRSE-H and TCRSE-L. In hospital GC, 17 cases $(3.11 \%)$ were EBV subtype, and 116 cases (21.21\%) were MSI subtype, both of which disclosed characterization of rich lymphocyte infiltration in tumor microenvironment and mixed type histology of Lauren classification. By analyzing GEO data with therapy record of nivolumab, an immune-checkpoint inhibitor, the sensitive patients revealed elevated levels of CD3Z, CD8B, FOXP3, IDO1 and PD-L1 in tumor. Conclusions: the present study proposed new predictive signature, a four-T cell combination for predicting sensitivity to immune-checkpoint inhibitor.

Keywords: Gastric cancer; Immune signature; Therapeutic response; Immune checkpoints inhibitor; Hospital biobank

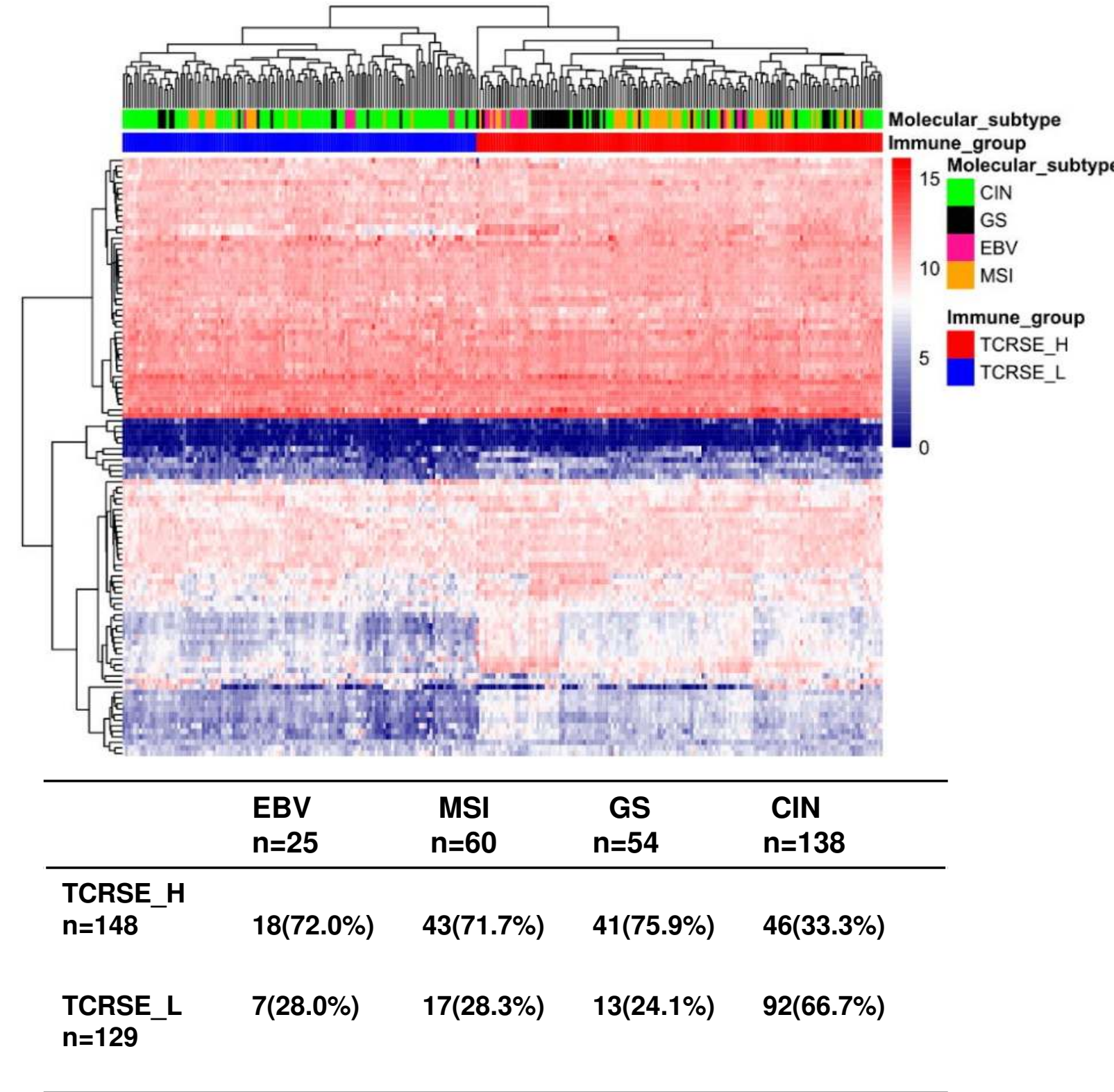

Based on KEGG T cell receptor signal pathway analysis, GC could be divided into TCRSE-H and TCRSE-L. TCRSE$L$ is well corresponded to EBV, MSI and partial GS subtypes, while TCRSE-L is corresponded to CIN subtype.
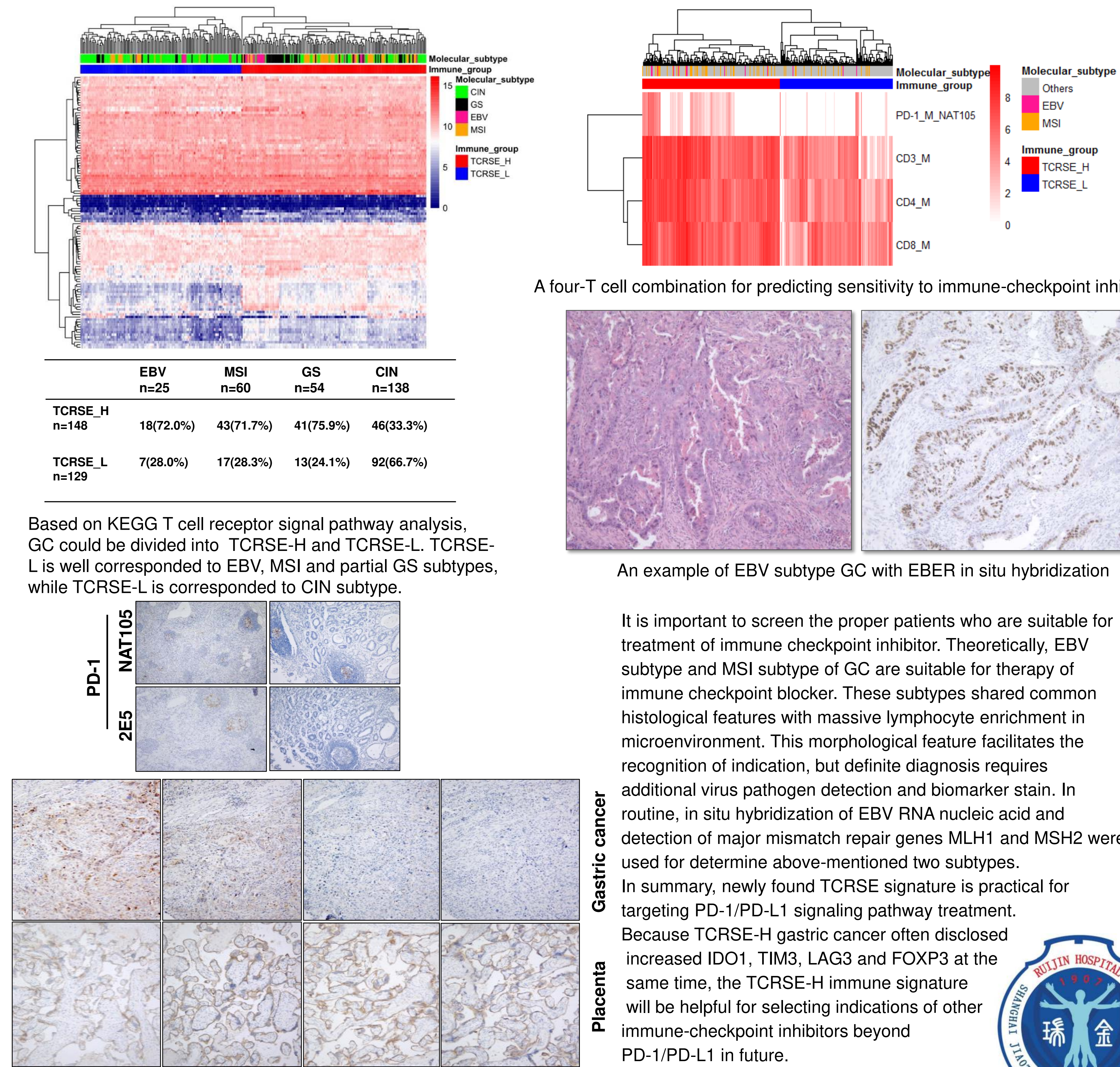

A four-T cell combination for predicting sensitivity to immune-checkpoint inhibitor

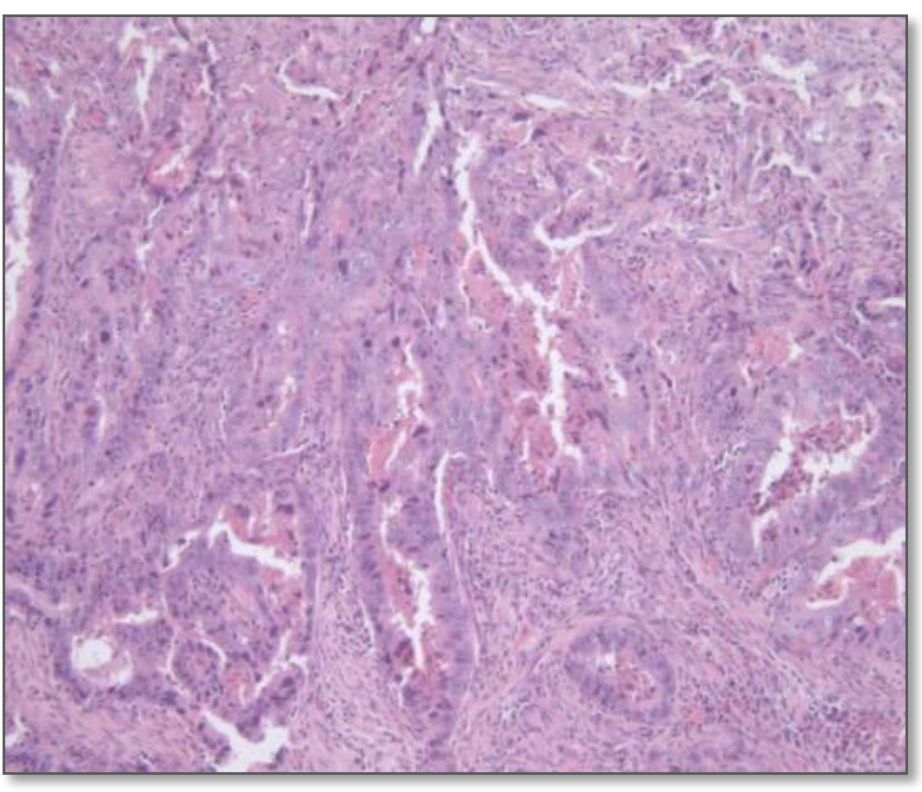

An example of EBV subtype GC with EBER in situ hybridization

It is important to screen the proper patients who are suitable for treatment of immune checkpoint inhibitor. Theoretically, EBV subtype and MSI subtype of GC are suitable for therapy of immune checkpoint blocker. These subtypes shared common histological features with massive lymphocyte enrichment in microenvironment. This morphological feature facilitates the recognition of indication, but definite diagnosis requires additional virus pathogen detection and biomarker stain. In routine, in situ hybridization of EBV RNA nucleic acid and detection of major mismatch repair genes $\mathrm{MLH} 1$ and $\mathrm{MSH} 2$ were used for determine above-mentioned two subtypes. In summary, newly found TCRSE signature is practical for targeting PD-1/PD-L1 signaling pathway treatment. Because TCRSE-H gastric cancer often disclosed increased IDO1, TIM3, LAG3 and FOXP3 at the c. same time, the TCRSE-H immune signature क् will be helpful for selecting indications of other immune-checkpoint inhibitors beyond PD-1/PD-L1 in future. 